\title{
NUEVOS RETOS Y OPORTUNIDADES DE MEJORA EN LA LUCHA CONTRA INCENDIOS FORESTALES Y SUS CONSECUENCIAS
}

\author{
José Antonio Vega Hidalgo \\ Centro de Investigación Forestal de Lourizán. Consellería do Medio Rural e do Mar. Xunta de Galicia. \\ Ado. 127.36080-PONTEVEDRA (España).Correo electrónico: jose.antonio.vega.hidalgo@ xunta.es
}

El fuego es un problema en el que la actitud objetiva no ha podido realizarse jamás. Gaston Bachelard (Psicoanálisis del fuego, 1938)

\section{Resumen}

Los incendios forestales suponen un desafío formidable a nuestra sociedad. A pesar de todo nuestro desarrollo tecnológico nos enfrentamos a un problema de magnitud global que lejos de remitir, continúa demandando cuantiosos medios para su control, muestra un elevada capacidad para generar alarma en las poblaciones afectadas, produce impactos económicos y ecológicos considerables y contribuye al aumento de los gases de efecto invernadero. Al mismo tiempo, el fuego es un factor ecológico esencial en multitud de ecosistemas. El incendio forestal aparece como un fenómeno de gran complejidad con multiplicidad de facetas, en donde se superponen e interaccionan factores socioculturales, políticos, urbanísticos y económicos, junto a físicos, ecológicos, medioambientales, tecnológicos y de seguridad, entre otros muchos. Todos ellos hacen muy difícil la tarea de la "gestión del fuego". Nuevos retos han surgido en los últimos años, o han cobrado nueva fuerza, como los problemas asociados a la interfaz urbano- forestal, la acumulación de los combustibles forestales, los efectos negativos del cambio climático, la creciente incidencia de la combustión de biomasa en los gases de efecto invernadero, la crisis económica, entre otros. Pero ofrecen también ocasión para desarrollar mejores sistemas de protección contra incendios, aumentar la eficiencia de los medios de lucha, incrementar la seguridad de los combatientes y de la población amenazada, armonizar los objetivos y medios de la gestión forestal sostenible con los de protección de incendios, expandir el aprovechamiento de la biomasa con fines energéticos, evaluar, con más precisión, las situaciones de riesgo pre y post-fuego y recuperar mejor las áreas afectadas. Estamos, sin embargo, lejos de lograr un equilibrio entre el control de los fuegos no deseados y el reconocimiento del importante papel jugado por los regímenes de fuego en el mantenimiento de la biodiversidad de nuestros ecosistemas forestales y la utilización de ese hecho en la gestión. En ese contexto, la investigación científica y técnica sobre el fuego forestal constituye una poderosa herramienta que ha permitido, a lo largo de los últimos decenios, incrementar sustancialmente nuestra compresión del fenómeno, apoyar tecnológicamente la protección frente a él y, lo que es más importante, desarrollar nuevas perspectivas para abordar los diversos problemas asociados. En las líneas que siguen se reflexiona sobre algunos de los interrogantes planteados en torno al problema de los incendios y se sugieren vías de investigación para responder a esos desafíos.

Palabras clave: Montes, Restauración, Investigación, Medios de Lucha 


\section{INTRODUCCIÓN}

A pesar del empleo, cada vez más pronunciado, de numerosos y complejos medios tecnológicos en la protección contra incendios forestales, y de los cuantiosos recursos utilizados para protegerse de ellos, siguen constituyendo cada año una amenaza potencial, en muchas partes del mundo, entre ellas nuestro país, con capacidad para provocar alarma en la sociedad y fuertes impactos económicos y ecológicos. Retan así nuestro aparente dominio de la naturaleza, recordándonos nuestras limitaciones para ejercer algún tipo de control sobre muchas de sus manifestaciones.

El incendio se nos revela como un fenómeno complejo, a escala global, presentando multiplicidad de facetas, en donde se superponen e interactúan numerosos aspectos socioculturales, políticos, y económicos, junto a físicos, ecológicos, medioambientales, tecnológicos y de seguridad, entre otros (FAO, 2007; FlanNIGAN et al., 2009). Plantea también, en toda su crudeza, las enormes diferencias socioculturales y económicas existentes en el mundo y las distintas percepciones y maneras de relacionarnos con el fuego (PyNE, 1995). Todo ello conlleva enfrentarse a numerosos interrogantes, lo que hace muy difícil la tarea de la "gestión del fuego". Con frecuencia el incendio es considerado por la sociedad urbana y los medios de comunicación del primer mundo como una "emergencia", una cuestión de seguridad, y ciertamente puede serla en muchas ocasiones, pero esa visión oculta muchos otros componentes del problema y contrasta fuertemente con la del hombre rural. Esas diferencias de percepción se agigantan cuando comparamos la actitud frente al fuego del mundo desarrollado económicamente con la de los países en vía de desarrollo.

Tenemos por delante una intensa tarea, en términos de mejora del conocimiento científico, y del desafío de compatibilizar ese conocimiento con la reducción de los impactos socioeconómicos de los incendios que consideramos negativos parar el hombre, al tiempo que mejoramos también de los sistemas de rehabilitación y restauración de las áreas forestales afectadas y aprovechamos esa oportunidad para crear paisajes más resistentes y resilientes al fuego.
Hay que prestar también atención a nuevos factores condicionantes del incendio que han aparecido en escena, se han agravado en los últimos años, o pueden hacerlo en el futuro. Suponen retos a nuestra capacidad científica y técnica, y, al mismo tiempo, se mezclan con los factores socioeconómicos que, lejos de remitir, siguen presentes, como un marco general del problema. En las líneas que siguen se comentan algunos de los problemas mas relevantes asociados a esos escenarios a los que debería dirigirse una investigación al servicio de la sociedad.

\section{NECESIDAD DE UN CAMBIO COLECTIVO DE MENTALIDAD Y DE UNA INTEGRACIÓN DE PARADIGMAS SOBRE EL FUEGO}

Uno de los retos más importantes en relación con los incendios forestales es el de lograr que se produzca un cambio de percepción y de mentalidad respecto al complejo papel que el fuego juega en los sistemas forestales, tanto por parte de los profesionales involucrados en la protección contra incendios como por la sociedad en su conjunto, con particular énfasis en los grupos ambientalistas y medios. La transferencia de la investigación sobre el fuego forestal no ha sido fácil hasta ahora en el contexto europeo. Este problema no es exclusivo de nuestro ámbito. Durante mucho tiempo se ha contemplado al incendio forestal como un "accidente", con la connotación no solo de "perturbación," de un equilibrio preexistente, que eso conlleva, cuando no de desastre (PAUSAS et $a l ., 2008$ ), sino incluso como algo atípico y ajeno a los ecosistemas forestales. Esta última visión, que procedía de la selvicultura alemana, poco familiarizada con el fuego en los ecosistemas centroeuropeos, y defensora de su exclusión, fue exportada a las Escuelas Forestales de los países mediterráneos, de Estados Unidos y de otros, y dio lugar, durante muchos años a una visión errónea del fuego forestal y a una falta de inclusión de esta materia en la universidad. Está detrás de una política de protección contra incendios basada casi exclusivamente en la extinción del fuego, con prohibiciones muy severas respecto a su uso. Esa aproximación ha sido ampliamente cuestionada desde la investigación (e.g. 
Conrad, 2001; Fernándes, 2008; Moreira et $a l ., 2011)$. Sin embargo, es muy difícil sustraerse a ella. Cuando las igniciones son muy numerosas, hay un incendiarismo pronunciado y la sociedad urbana exige la eliminación de los fuegos, los responsables políticos no tienen fácil cambiar de estrategia. Como contraste, en Estados Unidos y Australia la política de "contar" con el fuego ha impregnado la gestión de incendios desde la década de los setenta (e.g. PYNE et al., 1996; GILL, 2005; DonOvan \& Brown, 2007), aunque eso tampoco quiere decir que el problema se haya solucionado. En nuestro país y los de nuestro entorno próximo, la utilización del fuego prescrito es frecuentemente invocada (e.g. DíAz Delgado et al., 2004; PiÑol et al., 2007; FERNÁNDES, 2008; DUGUY et al., 2007), especialmente después de grandes fuegos catastróficos. Pero esto no se ha traducido en una mayor utilización de esa técnica. Frente a este hecho, la gestión integrada del fuego, en el sentido de MYERS et al. (2006) representa una aproximación más global al fuego, que trata de evaluar y equilibrar las amenazas planteadas por el incendio con sus beneficios, al considerar los contextos medioambiental, socioeconómico cultural y político del fuego. Esta propuesta, trata de armonizar los conocimientos científicos las necesidades sociales y las tecnologías de la gestión del fuego a múltiples niveles. El reciente proyecto europeo FIREPARADOX (http://www.fireparadox.org) ha perseguido desarrollar y transmitir esa idea.

Estrechamente conectado con esta misma cuestión se encuentra el desafío de la falta de integración de los tres paradigmas principales sobre el fuego forestal que no solo mantiene impregnada la investigación científica sobre el tema desde su origen, sino que ha trazado caminos que parecen cada vez más divergentes. Según Pyne (2007) esas tres visiones parten de considerar al fuego como un fenómeno casi exclusivamente físico, biológico y humano, respectivamente, y haber sido construidas como completas en sí mismas. Según el autor, el predominio del paradigma físico ha venido apoyado por ser el primero en surgir y estar dirigido a la extinción del fuego, un requerimiento de la sociedad urbana y de los responsables políticos. Sin embargo, se necesita una aproximación holística a la investigación sobre el fuego fores- tal (Bowman et al., 2009) ya que el fuego no puede ni debe ser excluido de esos ecosistemas propensos a él porque seguimos necesitándolo para el manejo del territorio y el mantenimiento de los ecosistemas (Burrows, 2008; Pausas \& KeEley, 2009). Tenemos, como dicen MYers et al. (2006) y BIROT (2009) que aprender a convivir con el fuego y, como señala HIRSCH (2001), de la forma más inteligente posible.

\section{RÉGIMEN DE FUEGO}

Una mejor comprensión del régimen de fuego resulta esencial para evaluar el papel que el fuego juega en los ecosistemas forestales, fundamentar una gestión de la vegetación y usos del territorio que incida favorablemente en el problema de los incendios, y de adaptación y mejora de la resiliencia al cambio climático, entre otros importantes beneficios prácticos. Por otro lado, el estudio del régimen de fuego ofrece una buena oportunidad para ayudar a conciliar las tres aproximaciones investigadoras que han elaborado paradigmas sobre el fuego, y que hacen muy difícil establecer un marco común para su estudio y para encontrar soluciones a las demandas y necesidades sociales sobre los incendios forestales.

Se admite que el régimen de fuego tiene como agentes conductores el clima, la vegetación y la acción del hombre (JOHNSON, 1992).

¿Por qué resulta relevante la determinación de la importancia relativa de esos agentes? Básicamente, porque ello fundamentaría mejor nuestra estrategia de protección contra incendios. Por ejemplo, PIÑol et al. (2005) se plantean: Si los combustibles son los agentes controladores más importantes del régimen de fuego, la estrategia de exclusión total de fuego, y su corolario, el reforzamiento de la actividad de supresión como eje principal de la gestión del fuego, tenderían a promover la aparición de grandes incendios por acumulaciones crecientes de combustibles y los tratamientos de éstos podrían ser útiles herramientas para la protección. Por el contrario, si los grandes incendios son el resultado de algunos eventos meteorológicos extremos, ni la exclusión del fuego ni los tratamientos, afectarían al tamaño de los incendios. 
Sin embargo, clarificar cuál de esos tres agentes mencionados es más influyente en cada sitio no resulta tarea fácil. PYNE et al. (1982) y ALLEN (2002), entre otros, nos han recordado decenios de discusiones sobre ese tema para los bosques estadounidenses del Oeste. Sin embargo, el debate está lejos de cerrarse (e.g. KEELEY \& ZEDLER, 2009; CARY et al., 2009; KRAWCHUK \& Moritz, 2011). Suele aceptarse que el clima tiene más peso cuando la densidad de población es baja, o genera un impacto pequeño (MARLON et $a l ., 2008)$, o las condiciones climáticas son muy pronunciadas (ARCHIBALD et $a l .$, 2009). Por contra, los defensores de la importancia de los combustibles (e.g. Minninch, 1983, 2001; MinNINCH \& CHOU, 1997) argumentan que los grandes incendios son un moderno resultado del éxito de la extinción (una paradoja de ese éxito). Otros sostienen que los grandes fuegos ocurren bajo condiciones meteorológicas extremas, y que entonces el fuego se propagará por todo tipo de vegetación, independientemente de la cantidad de combustible disponible (e.g. MorITZ ,1997; KeELEY et al., 1999; MoRITZ et al., 2004). Por otro lado, el hombre puede actuar sobre el régimen de fuego a través de tres medios: el control de la frecuencia de igniciones, la supresión de los fuegos y el uso del territorio, con sus efectos asociados sobre la cubierta vegetal y, en consecuencia, sobre la estructura y fragmentación de los combustibles.

En nuestro país el tema también ha sido y continúa siendo abordado, con conclusiones a veces no coincidentes. VÁZQUEZ et al., (2002), destacaron el papel crucial del hombre para cambiar las pautas espaciales de los incendios. Posteriormente (VÁZQUEZ et al., 2006) resaltaron el papel de los combustibles en el régimen de fuego. VÁZQUEZ (2012) confirmó la escasa explicación que el tipo de vegetación aportaba a la variabilidad de las pautas espaciales del citado régimen. Sin embargo, VIEDMA (2008) y MORENO et $a l$. (2011), en la zona Centro, observaron que la estructura de la vegetación tenia una apreciable influencia en el tamaño de los incendios

Por su parte, PIÑOL et al. (2005; 2007), encontraron, mediante simulación, que las hipótesis, del combustible y el clima, eran ambas compatibles con sus resultados. El área quemada total resultaría ser principalmente una fun- ción del clima y las características de la vegetación y eso no podría ser fácilmente cambiado por la estrategia de gestión. De hecho, el reforzamiento de las capacidades de extinción y el tratamiento de combustible no haría variar el área quemada anual, aunque en el primer caso aumentaría la proporción quemada por grandes incendios. Esto apoyaría la tesis de que la acumulación de combustible es el agente conductor del régimen Además, según estos autores, la intensidad de los incendios aumentaría con la supresión y disminuiría con los tratamientos, lo que justificaría la estrategia de tratamientos de combustible. Al mismo tiempo, el porcentaje de grandes incendios podría ser reducido, pero no eliminado con los tratamientos de combustible.

Una retroalimentación entre reducción del combustible y homogeneización del paisaje ha sido destacada como un factor controlando el régimen de fuego del $\mathrm{NE}$ de la península (LOEPFE et al., 2010). Las cubiertas con alta carga de combustible y el terreno homogéneo favorecen la propagación del fuego y éste reduce el combustible, transformando el bosque en matorral. Sin embargo, también incrementa la homogeneidad de combustibles, creando un feedback positivo a la propagación de nuevos incendios, más frecuentes en áreas ya quemadas.

En nuestro país, donde la influencia humana es tan antigua y marcada y las condiciones climáticas son relativamente moderadas, algunos autores estiman que el hombre es el factor más influyente en el régimen de fuego en esta región (LOEPFE et al., 2012; PAUSAS \& FERnÁNDEZMuÑoz, 2012). Al mismo tiempo, PAUSAS Y PAUlA (2012) sostienen que, bajo las condiciones mediterráneas, la estructura del combustible es más relevante en la actividad del fuego que la frecuencia de las condiciones climáticas que conducen a éste. Esta idea conecta con el modelo conceptual de KRAWCHUK \& MORITZ (2011) que ellos denominan "la hipótesis de los limitantes variables" de la actividad global de fuego, un marco que considera explícitamente gradientes espaciales en la importancia relativa de los agentes controladores de los incendios, más que una visión binaria de la meteorología/ humedad del combustible contra la cantidad de combustible. En particular, estos últimos autores proponen que la influencia relativa de esos agentes cambia 
de un lugar a otro, de acuerdo su escasez o variabilidad: donde los combustibles existen en cantidad disponible todo el tiempo, es probable que las condiciones de humedad del combustible sean relativamente más importantes (meteorología/clima); en contraste, si los recursos son efímeros, entonces la cantidad debería regular la frecuencia de los incendios (combustible).

Aunque se han desarrollado modelos que simulan características del régimen de fuegos (e.g. CARY, 1997; LI, 2000; KEANE et al., 2006; PERERA, 2008), habitualmente no incluyen la acción del hombre como un agente conductor del régimen de fuego (Moulliot \& Field, 2005). Mas recientemente, LOEPFE et al. (2011), han propuesto un modelo que también considera datos explícitos de la influencia humana, a través de la frecuencia de igniciones, la supresión del fuego y los cambios de uso del territorio. La modelización de las pautas espaciales de ignición es una tarea compleja (e.g. VEGA \& CHUVIECO, 2006; Romero-CALCERRADA, 2008; CHUvieco et al., 2010) ya que el comportamiento humano en relación al fuego es extremadamente difícil de modelar (FlanNigan et al., 2009; CONEDERA et al., 2011).Esto constituye un importante desafío para la predicción del régimen de fuego.

A pesar de los avances obtenidos en este campo, y del desarrollo de modelos con información espacial, subsisten importantes dificultades limitando nuestro conocimiento sobre el régimen de fuego. Uno de los problemas mas complejos es el de la reconstrucción de los regimenes pasados. También existe mucha incertidumbre respecto a la capacidad de los modelos de reflejar adecuadamente los efectos del cambio climático.

\section{EL CONTEXTO SOCIOECONÓMICO}

Desafortunadamente la sociedad no ha podido encontrar todavía más que soluciones parciales para la mayor parte de los denominados "factores estructurales" que subyacen en un elevado porcentaje de los incendios, ligados a escenarios socioeconómicos, educativos y culturales bien definidos y en un marco de condiciones climáticas favorables a la combustión de la vegetación.
Es muy revelador que si se lanza una ojeada al mapa de frecuencia de incendios en Europa y en nuestro país (ej. SAN Miguel y CAMIA, 2009; MoReno \& ChUVIECO, 2012) se observa cómo los territorios con un más elevado número de incendios coinciden, en bastante proporción, con las zonas más deprimidas, en términos de los factores mencionados. El solape es todavía mayor cuando la proporción de incendios de causa desconocida o intencionada crece. Mientras esas cuestiones estructurales no se aborden en profundidad será difícil que los incendios disminuyan de gravedad. Afortunadamente, el mapa de frecuencia de incendios no es muy coincidente con el de los que alcanzan grandes dimensiones, usualmente originados por causas diferentes a las anteriores, con períodos de recurrencia bastante más largos que los arriba citados, generalmente asociados a eventos meteorológicos desfavorables y grandes continuidades de combustible.

Modificar las condiciones socioeconómicas de la población en las áreas de mayor actividad de incendios es un verdadero reto para la sociedad en su conjunto, y sin duda para la investigación científica. En resumen, tenemos por delante un desafío pendiente de resolver, una cuestión que afecta a muchos más aspectos que el de la mera extinción de los fuegos no deseados. Un problema que consume recursos que podrían destinarse a otros cometidos, entre ellos forestales.

MoReIRA et $a l$. (2011), revisando la información de numerosos autores, han concluido que los cambios en el uso del territorio en las últimas décadas han incrementado la frecuencia de incendios y modificado notablemente la estructura de los combustibles en la cuenca mediterránea. La mayor frecuencia de incendios ha promovido paisajes más homogéneos, cubiertos de matorrales más propensos al fuego En algunas zonas los grandes incendios se han vuelto más frecuentes.

Para estos autores, los cambios socioeconómicos ocurridos serían, en última instancia, los responsables de ese cambio en la estructura de los combustibles. Esto nos lleva a la reflexión de PyNE (2007) en relación a si la política de los poderes públicos respecto a los incendios forestales y la investigación científica están dirigidas adecuadamente sobre esta problemática. El 
componente cultural y humano del fuego está siendo olvidado. El hombre es lo que es gracias al fuego y hoy ese hombre se ha hecho urbanita, perdiendo su conexión con el mundo rural, y ve al fuego como algo que puede amenazar sus bienes y frente al que solo adopta una postura de eliminación. Además, los enfoques físicos, tecnológicos y ecológicos del problema de los incendios forestales son demasiado absorbentes de recursos, y, hasta ahora poco reconciliables.

La creación de políticas que mejoren la calidad de vida en el ámbito rural manteniendo las actividades agrarias en sitios de prioridad para la reducción de combustibles, legalizando y promoviendo el uso del fuego prescrito, la actividad pastoral y el aprovechamiento de la biomasa con fines energéticos ha sido propuestos por bastantes estudios (ver MoreirA et al., 2011). Hay que reseñar varios importantes pasos dados en nuestro país en esa dirección que suponen un notable cambio de mentalidad sobre esta temática. La creación de los EPRIF, promovida por el Area de Defensa Contra Incendios Forestales del MAGRAMA, está dando numerosos frutos, en el ámbito de la prevención, al disponer de grupos de técnicos que trabajan en contacto con las necesidades rurales ligadas con el fuego, empleando el fuego prescrito y otros tratamientos alternativos de combustibles (VÉLEZ, 2002, 2005, 2010). Este programa, cuenta con un soporte científico que está evaluando algunos aspectos relacionados con los posibles efectos ecológicos de esos tratamientos, sobre la vegetación y el suelo. El programa 42 de la Junta de Castilla León ha sido otra iniciativa relevante en esa misma línea de prestar un servicio conectado directamente con el mundo rural y dirigido a encauzar un problema grave de causalidad de incendios. En igual sentido han operado otras acciones, como el progresivo aumento de la utilización del ganado para control del combustible vivo, iniciada en el CIF de Lourizán, en los ochenta y más recientemente desarrollada en el CSIC (RuIZ-Mirazo, 2009, 2011) e implementada por la Junta de Andalucía, Comunidad Valenciana y en Cataluña, así como en el SO de Francia (ETIENNE et al., 1996; ThAVAUd, 2009).

El proyecto FIRESMART (http://www.firesmart-project.eu/private.action) ha efectuado un análisis DAFO de la situación de la prevención de incendios forestales en España (HERNANDO et al., 2012), destacando la necesidad de acometer acciones integradas para reducir el riesgo estructural, conectadas con la gestión forestal sostenible, que involucren a los propietarios y gestores forestales y dotándolas de mecanismos de financiación. También proponen el fomento del empleo rural y la mejora de las condiciones socioeconómicas del sector, al tiempo que se valorizan sus productos y servicios. Desarrollar estrategias de comunicación, concienciación y formación dirigidas a grupos específicos, estimulando su participación, y sostenidas en el tiempo son también otras propuestas y oportunidades de mejora.

Desde la perspectiva de investigación se requiere profundizar en la determinación del las causas de las igniciones de origen humano, lo que implica ahondar en las raíces culturales, sociales y económicas de los incendios. Sin duda, esta investigación tiene también que conjugarse con medidas de política agraria y acciones de concienciación y educación y en su caso también medidas legales coercitivas.

\section{CAMBIO CLIMÁTICO}

En los últimos años las posibles consecuencias del cambio climático sobre los incendios forestales están recibiendo una gran atención por parte de la investigación en todo el mundo, incluida España (véanse, p. ej. las revisiones de Moreno et $a l .$, 2005, FlanNigan, 2009). En el ámbito europeo, el proyecto FUME - Forest fire under climate, social and economic changes (www.fumeproject.eu), actualmente en desarrollo, es un buen ejemplo de acción integrada dirigida a diseñar estrategias adaptativas. No hay que olvidar que el incendio forestal es también un factor coadyuvante al cambio climático. Es muy probable que las interacciones entre el cambio climático y los cambios socioeconómicos generen un marco en donde muchos de los aspectos cruciales de la protección contra incendios como el riesgo, el comportamiento del fuego, la severidad del mismo, la vulnerabilidad de los ecosistemas, los impactos causados en ellos por el fuego y las estrategias de prevención, combate y restauración forestal de áreas quema- 
das ó no, se vean profundamente alterados. De hecho, se requerirá un importante y continuado esfuerzo de adaptación, a ese nuevo escenario, de las políticas de protección contra incendios, incluyendo su prevención, la extinción, y la restauración posterior. También los planteamientos técnicos y la gestión de esos componentes, necesitará de nuevos enfoques, acordes con esa situación. Toda esa labor constituye un reto de gran magnitud para el que se necesitará no solo un incremento sustancial de la investigación en muchos campos, sino lo más importante, una verdadera interdisciplinariedad dada la gran complejidad de los problemas abordar.

Los estudios de ámbito nacional y regional en la península ibérica han encontrado hasta ahora, generalmente, relaciones significativas entre variables o índices de peligro de base meteorológica y actividad de incendios, aunque no en todas las regiones (PIÑOL et al., 1998; Pausas, 2004; Vega et al., 2009; Carvalho et al., 2010; Moreno et al., 2010). Han evidenciado también un incremento de su intensidad y severidad en los últimos decenios en algunas regiones de nuestro país.

En términos generales, se espera un incremento del riesgo de incendios en el área medite- rránea (Moriondo et al., 2006; AlCAMO et al., 2007; LIU, 2010). Sin embargo estos trabajos no han considerado los posibles cambios en la vegetación (combustibles), en la influencia del hombre sobre el régimen de fuego, que el cambio climático puede inducir, ni tampoco las interacciones ciertamente complejas que pueden surgir (HessL, 2011). Todo ello significa que existen muchas incertidumbres, particularmente en el medio y largo plazo. Uno de los aspectos de más difícil estimación es cómo afectará el cambio a la vegetación. Aunque en términos generales se pronostica un aumento de la vegetación más adaptada a la sequía (AlCAMo et $a l ., 2007$; DURY et $a l ., 2011)$ en el área mediterránea y en gran parte de nuestro país, esto puede venir acompañado de disminución de la cobertura vegetal y la productividad primaria en otras grandes (LINDNER et al., 2010; GRACIA et al., 2005).

La Figura 1, modificada de FERnANDES (2013), esquematiza los principales efectos esperados del cambio global sobre los elementos del régimen de fuego en sistemas dominados por las igniciones de origen humano, y es, en sí misma, un buen exponente del elevado grado de incertidumbre existente sobre la cuestión. Las principales carencias de conocimiento provienen del

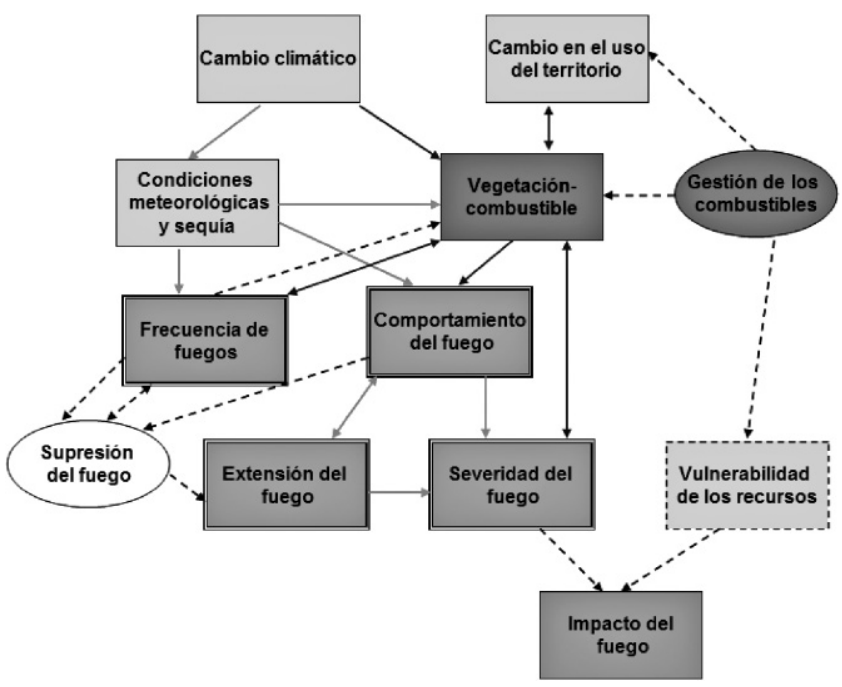

Figura 1. Efectos del cambio global sobre elementos del régimen de fuego y de la gestión de los combustibles. Modificado de Fernandes (2013). Las líneas en rojo indican efectos que refuerzan condiciones mas desfavorables para los incendios. Las líneas en azul representan efectos sobre los que existen incertidumbre sobre su influencia en el régimen de fuego. Las líneas de puntos indican efectos de las acciones sobre los combustibles 
entendimiento insuficiente de la importancia relativa de los combustibles y condiciones meteorológicas y sequía sobre el régimen de fuego. Asimismo, la conexión entre los procesos del cambio global y el régimen de fuego no es directa, y nos está bien establecida (FERnANDES, 2013). Estos desconocimientos se trasladarán inevitablemente a las propuestas de supresión y de gestión técnica de los combustibles forestales.

Un reciente trabajo (LOEPFE et al., 2012) ha estimado, mediante simulación, los impactos del cambio climático y de diferentes estrategias de gestión de los incendios (aumento de la capacidad de los medios de extinción, disminución de las igniciones y aumento de la superficie de cultivos agrícolas), en el NE de España, bajo diversos escenarios de cambio climático, durante el siglo XXI. Sus resultados indicarían un drástico incremento del área anual quemada, menos pronunciado en sitios más secos, y un riesgo de inesperados grandes incendios catastróficos, aparentemente apoyando la hipótesis de PUEYo (2007). Esas estrategias podrían reducir, pero no compensar totalmente, los cambios inducidos en el régimen de fuego. La más efectiva combinaría una gestión llamemos "tradicional", basada en una reducción de las igniciones y el reforzamiento de la supresión, junto a la propuesta de creación de campos agrícolas en áreas marginales, estratégicamente dispuestas, que reducirían la extensión del área quemada. Aunque los autores no detallan la realización de sus propuestas, resulta claro que la solución no es sencilla. Por otro lado habría que preguntarse si esos paisajes rurales que se evocan, pueden reconstruirse y si tienen viabilidad en nuestra sociedad actual, pero eso sería otro reto. Es claro que necesitamos refinar más los modelos actuales (THONICKE et al., 2010; FERNANDES, 2013).

\section{LA GESTIÓN DE LOS COMBUSTIBLES}

Los problemas asociados a la gestión de los combustibles implican principalmente cinco desafíos: a) modificar el régimen de fuego, b) reducir la severidad del fuego y sus daños asociados más que el tamaño de los incendios, c) efectuarla en sintonía con la adaptación al cambio climático, d) consiguiendo una mejor eva- luación de los efectos de los tratamientos de los combustibles y e) integrándola en una actividad forestal sostenible, en el marco de una gestión multiuso y ecológicamente sensible del territorio.

Actuando sobre los combustibles podemos influir en un elemento clave en el régimen de fuego, disminuyendo el área quemada, de una forma directa, al afectar a la velocidad de propagación del fuego, y hacer a los sistemas de extinción más eficientes y seguros. Indirectamente, al generar condiciones microclimáticas más favorables y especialmente podemos influir en la severidad del incendio y por tanto en su impacto medioambiental.

El comportamiento del fuego se ve afectado por las características de los combustibles, particularmente su estructura. Sin embargo, no debemos esperar una panacea con los tratamientos de combustibles (REINHARDT et $a l .$, 2008). Todas las estrategias, desde el aislamiento por áreas cortafuegos, pasando por la modificación en la estructura, continuidad y carga de los combustibles en áreas, hasta la conversión de la vegetación a tipos de combustibles menos peligrosos pueden tener un papel positivo en dificultar la propagación del fuego, aunque también presentan limitaciones (e.g. Perchat \& Rigolot, 2005; SYPHARD et $a l .$, 2011).En general, las reducciones de combustible son acordes con las recomendaciones selvícolas de adaptación del bosque mediterráneo al cambio climático (GRACIA et $a l ., 2005$; SERRADA et $a l .$, 2011). La conversión de tipo de bosque, particularmente la transformación de masas de coníferas a frondosas es frecuentemente invocada como una solución para los grandes incendios, y ciertamente sería deseable aumentar nuestra superficie de caducifolias, estrictamente desde un punto de vista de protección contra incendios. La cuestión es si ello es factible, con los condicionantes climáticos, ecológicos y socioeconómicos que tenemos. Las masas de frondosas, especialmente las caducifolias, suelen ser más efectivas para modificar el comportamiento del fuego y restringir su propagación a escala de paisaje, en comparación con otras formaciones (e.g. DIAZ DELGADO et al., 2004; GONZÁLEZ et al., 2006; Fernandes et al., 2010; PEREIRA \& Vicente, 2010; Moreno et al., 2011). Pero el tema es más 
complejo, porque la estructura de la masa, afecta a los combustibles y a la meteorología local. De esta forma las características de las masas pueden amortiguar o favorecer el efecto de la composición del bosque sobre el comportamiento del fuego (FERNANDES, 2013). Parece razonable, empezar por mantener en un buen estado las masas de planifolias que tenemos y dirigir la evolución de muchas de las repoblaciones que existen y en donde las planifolias están presentes hacia formaciones mixtas.

Una cuestión de fondo en nuestro país sobre este tema es que desde ciertos sectores se han sacralizado las especies planifolias autóctonas y al mismo tiempo se ha llegado a negar carta de autoctonía a nuestros bosques de coníferas, considerados elementos extraños del paisaje, introducidos por el hombre y hasta responsables de los incendios. Es verdad que se ha promovido en la última mitad del siglo XX en los países del S de Europa un incremento de extensas y continuas superficies de vegetación particularmente inflamable, si bien en muchas de las áreas repobladas, existían dificultades ecológicas y técnicas para que otras especies prosperaran. Por otro lado, se dio preferencia a la expansión de la reforestación sobre los cuidados culturales. Pero la vegetación no arde sola. La sociedad no quiere asumir que tenemos un problema de fuerte incendiarismo y mal uso del fuego en muchas partes de país, y que los condicionantes socioeconómicos y climáticos y sus efectos en los combustibles están ahí.

Los nuevos escenarios de cambio climático y socioeconómico pueden volver al régimen de fuego más dependiente del clima y meteorología, con lo que es probable que disminuyan las oportunidades para que el régimen de fuego pueda ser modificado por la gestión de los combustibles. Su eficacia depende también de la proporción de territorio tratado, la frecuencia de aplicación y la velocidad de recuperación de lo combustibles (FINNEY et al., 2007; PRICE \& Bradstock, 2010; VILÉN y Fernandes, 2011). Cabe destacar que en este momento, los tratamientos de combustibles están recibiendo un fuerte impulso institucional en Estados Unidos (Millar et al., 2007), para restaurar la estructura del paisaje de forma que refleje su variabilidad histórica y promover ecosistemas más saludables frente al ataque de plagas y mejor adaptados al cambio climático. Abren también oportunidades para el aprovechamiento de los combustibles extraídos como fuente de energía (PRESTEMON et $a l ., 2012$ ), ayudando a reducir el coste de los tratamientos.

Un reto importante asociado a todos los tratamientos de combustible es el de evaluar su eficacia sobre el comportamiento del fuego en un posible incendio y sobre el régimen de fuego (e.g. Agee \& Lolley, 2006; Vaillant et al., 2009; Moghaddas et $a l ., 2010$ ), así como sus posibles efectos ecológicos, incluyendo su potencial para mitigar las emisiones de $\mathrm{CO}_{2}$ de los incendios (eg. NARAYAN et $a l, 2007$; AGER et al., 2010; HURTEAU et al., 2011; VILEN \& FERNANDES, 2011). Para ello necesitamos realizar una aproximación con fuegos experimentales, además del análisis de los incendios pasados y futuros y de simulación, mejorando previamente los sistemas de predicción del comportamiento y de efectos ecológicos del fuego.

Recientemente (HERNANDO et al., 2012, FERNANDES, 2013) se ha insistido en la necesidad de una gestión del combustible "inteligente" en el sentido de HIRSCH et al. (2001), esto es, una aproximación integrada, primariamente basada en los tratamientos de combustible, a través de los que se minimicen los impactos socioeconómicos, mientras se mantengan y maximicen los beneficios ecológicos. Básicamente, su objetivo sería promover paisajes mas resistentes a la propagación del fuego y ecosistemas más resilientes a los incendios (REINHARDT et al., 2008; Moritz \& StePHENS, 2008; STEPHENS et al., 2010). Se perseguiría dar prioridad a limitar la severidad de los incendios más que a la extensión quemada.

El manejo de fuegos no planeados, podría considerarse en un futro próximo como parte de esa estrategia proactiva, más compatible con el papel del fuego en modelar muchos de nuestros paisajes y en tratar de reducir fuertes severidades de incendios, indirectamente asociadas a la exclusión del fuego. Diferentes factores deberán ser tenidos en cuenta, porque el coste de la extinción, los posibles efectos ambientales adversos, el riesgo de escape para la población, tendrán que ser balanceados con sus ventajas ecológicas, dificultad de supresión y severidad de futuros incendios. 
Necesitamos resolver el problema de lograr una integración más efectiva de los tratamientos de combustibles en la selvicultura, en el marco de una gestión forestal sostenible y de una planificación multiuso del territorio, puesto que es a escala de paisaje donde las cubiertas vegetales influyen más en el incendio (STOCKMAN et al., 2010; LeHMKUHL et al., 2007). Lo cierto es que las actividades de selvicultura preventiva se han convertido en el motor principal de muchos proyectos de gestión forestal, en varios países. Pero no podemos considerar ese objetivo como excluyente de otros. Ni la selvicultura puede hacerse de espaldas al fuego, ni toda ella puede girar en torno al problema de los incendios. Finalmente la selvicultura también tiene que estar enmarcada en un cuadro más general.

\section{INTERFAZ URBANO-FORESTAL}

Paralela a los cambios socieconómicos ocurridos en la segunda mitad del siglo XX, ha surgido la creación de áreas urbanizadas en contacto con, o rodeadas por áreas forestales, constituyendo lo que se ha denominado la interfaz urbanoforestal (IUF). Se trata de asentamientos de población con una densidad notablemente más baja que la de las urbes, y usualmente incorporando vegetación junto a las edificaciones. Estas zonas presentan, generalmente, alta penetrabilidad al viento. La existencia de esta interfaz introduce factores nuevos en la prevención y extinción de incendios forestales. Las más importantes son un incremento notable del riesgo a las personas y edificaciones en estos incendios y una distracción de los medios de combate hacia la protección de esas áreas, con la consiguiente disminución en su eficacia y el incremento de la superficie forestal quemada. La evacuación de la población afectada añade complejidad a las tareas de supresión de este tipo de fuegos.

La tendencia general en los países desarrollados es la de incremento continuo de la IUF, sin un parejo aumento de las medidas de autoprotección individual ni de la exigencia de planes de protección y control de su ejecución (GILL \& STEPHENS, 2009; Moreira et al., 2011; Galiana et al., 2012). Este cuadro puede agravarse si las condiciones climáticas empeoran en el futuro y las limitaciones presupuestarias restringen las acciones preventivas. En algunos países más del 50\% de los presupuestos de tratamientos de combustibles se destinan a la IUF (MELL et al., 2010; QuAdrenNial Fire REVIEW, 2009).

Numerosos retos han surgido como consecuencia de la afectación de la IUF en los incendios forestales. Se precisa concienciar a la población potencialmente impactada por el incendio del riesgo al que está sometido y de la necesidad de realizar medidas de protección que deben ser comunitarias para que sean eficaces. Se requiere disponer de buenos sistemas de cartografiado de las IUF, cuantificar adecuadamente el riesgo de incendio y el grado de vulnerabilidad de las IUF (STEWARD, 2003; CAMIA et al., 2003; CABAllero, 2004; RADELOFF, 2005; Galiana et al., 2007; LAMPIN et al., 2006, 2009, 2010, 2011; MARTíNEZ et al., 2009). Es necesario también la elaboración de planes de defensa así como la exigencia y vigilancia por parte de la administración de la ejecución de las medidas de protección correspondientes. Recientemente MELL et al. (2010) han recogido las necesidades de investigación más importantes en este campo. Entre ellas cabe destacar la evaluación de la capacidad de los tratamientos de combustible para alterar el comportamiento del fuego, y la generación de pavesas y de humo en los incendios. Es asimismo urgente expandir el conocimiento sobre las condiciones de exposición al calor y pavesas que sufren las estructuras en la IUF, durante los incendios, De igual forma existe una necesidad apremiante de obtención de datos más precisos del comportamiento del fuego en incendios reales que permitan testar y mejorar los modelos de predicción del comportamiento. Ello redundará en más seguridad para los habitantes y en mejores decisiones respecto a la necesidad y el momento oportuno de la evacuación de las IUF.

\section{ECONOMÍA Y PROGRAMAS DE PROTECCIÓN CONTRA-INCENDIOS}

La ciencia económica es esencial para una correcta cuantificación del problema de incendios. De hecho, el análisis económico debe considerarse como una parte integral de una gestión más proactiva de los incendios forestales 
(MAVsAR et al., 2010). Hay dos cuestiones donde su aportación es particularmente relevante: en lo referente a la evaluación de los costes de los medios empleados en los programas de protección, y en la estimación, en términos económicos, de los efectos de los incendios sobre los recursos afectados. Juega asimismo un imprescindible papel en la búsqueda de soluciones adecuadas para optimizar la eficiencia de los recursos destinados a la protección (DONOVAN \& Rodeot, 2003; GonZÁleZ-CABÁN, 2007; MERCER et $a l .$, 2007). En un contexto de limitaciones presupuestaria para la extinción del fuego, de carácter global, cobra especial relevancia una eficiente utilización de los recursos de protección contra incendios. Las herramientas econométricas basadas en el análisis marginal permiten integrar los costes de extinción y el cambio neto en el valor de los recursos por efectos del fuego, dentro del marco presupuestario disponible y de acuerdo a determinadas filosofías de despacho de combinación de medios de extinción. En este sentido el modelo SINAMI (RoDRíGUEZ Y SILVA y GONZÁLEZ-CABÁN, 2010) y su programa informático ECONOSINAMI (RoDRíGUEZ Y SILVA, 2009), son un excelente ejemplo de esa aproximación. Conjugan el análisis estratégico de los presupuestos con la aportación de información de base para realizar estudios de tendencias presupuestarias, permitiendo así abordar predicciones de las necesidades de medios en marcos temporales determinados.

Los métodos econométricos no paramétricos, caracterizados por técnicas de análisis por envolvente de datos, están siendo utilizados en la determinación de la eficiencia de las operaciones de extinción. Estas técnicas, por semejanza con los sistemas productivos, determinan la eficiencia en el proceso de extinción del fuego a través de la relación existente entre los recursos empleados o asignados y el producto obtenido, de acuerdo a una meta establecida.

La valoración de daños y perjuicios provocados por los incendios forestales requiere del estudio individualizado de cada uno de los recursos tangibles (RodRíGUEZ y SILVA et al., 2012) e intangibles y el cambio del valor neto de los mismos en relación a la severidad del fuego y a la resiliencia del ecosistema (MolinA et al., 2009).
La consideración de la vulnerabilidad de los recursos afectados por el fuego en el ámbito de la evaluación de los daños y perjuicios producidos por los incendios ha ofrecido una nueva perspectiva en relación a la evaluación del impacto de los incendios sobre los recursos. Este concepto combina el valor del recurso, el impacto sobre él producido por el incendio, con el comportamiento del fuego. La integración de eso elementos se realiza mediante una matriz de ratios de depreciación en base a la intensidad del fuego (MolinA et al., 2011; RodRíGUEZ Y SILVA et al., 2012). Las herramientas de análisis econométrico como los modelos SINAMI y VisualSEVEIF (RodRíGUEZ Y SILVA et al., 2012), ofrecen, sin duda, oportunidades de análisis y diagnóstico de los paisajes y escenarios forestales que ayudan a definir los presupuestos bajo criterios de eficiencia. Este último modelo está basado en la integración de algoritmos que permiten determinar de forma geo-referenciada el impacto potencial del incendio, en términos de depreciación económica del valor de los recursos naturales, y con una dependencia directa del nivel de intensidad de fuego.

Todos los esfuerzos anteriores han permitido avanzar en una mejor utilización de los recursos de protección contra incendios y en una mas correcta evaluación del las pérdidas causadas por los incendios y de su efecto potencial en el bienestar de la sociedad. Estas aproximaciones deben completarse con la cuantificación económica de los efectos de diferentes medidas de protección contra-incendios sobre el riesgo, extensión y severidad de estos (PRESTEMON et al., 2008), y particularmente referidas a la interfaz urbano-forestal. (Mell et al., 2010).

\section{PREDICCIÓN DEL COMPORTAMIENTO DEL FUEGO}

Este es uno de los desafíos más importantes al que nos enfrentamos en el problema de los incendios forestales, junto con el cambio de pautas de ignición por parte de la población y la adaptación al cambio climático $\mathrm{Su}$ relevancia proviene del hecho de que afecta a casi todos los demás componentes del problema. Sin embrago, 
esto no es siempre bien entendido por los responsables de la protección.

A pesar de las destacadas contribuciones efectuadas en este campo por el desarrollo de modelos empíricos, semiempíricos y físicos y sus aplicaciones de base cartográfica (véanse revisiones de Weber (1991), PERrY (1998), PASTOR et al. (2003) y Sullivan (2009a,b,c), sigue siendo una necesidad acuciante disponer de mejores modelos predictivos del comportamiento del fuego. La irrupción de los modelos físicos tridimensionales de comportamiento del fuego ha supuesto un paso adelante científico muy considerable (LiNN et al., 2002; MELL et al., 2007). Un importante reto asociado a su utilización es lograr que trabajen en tiempo real en el incendio, siendo preciso también validar y testar sus predicciones con datos reales. Estos modelos requieren una caracterización espacial de los combustibles con mucho detalle, implicando mejorar los sistemas de inventario y cartografiado actuales.

Algunas de las muchas carencias y requerimientos de mejora en la predicción del comportamiento del fuego se esbozan seguidamente. Es necesario:

a) Incrementar nuestro conocimiento de la interacción combustible-fuego-meteorología a nivel local y de paisaje, para fundamentar mejor los modelos de propagación y la eficacia de los tratamientos de combustible. Se requiere una aproximación a dos escalas de las características de los combustibles: a nivel macro, donde la teledetección y la tecnología LIDAR han supuesto un avance muy considerable en ese objetivo, (RIAÑo et $a l$., 2004; ERDODY \& MosKaL, 2010) y micro, ya que los modelos nuevos de comportamiento son mas demandantes de información sobre combustibles (PARSONS et al., 2011).

b) Disponer de una mayor fiabilidad en la predicción meteorológica ligada al fuego, particularmente del viento, su interacción con la topografía y su influencia sobre el incendio. (WERTH, 2011; Clements, 2011; Sharples, 2009) y su influencia en la humedad de los combustibles.

c) Mejorar los sistemas de predicción de emisión de pavesas y generación de focos secundarios (GUIJARRO et al., 2002, 2004; GANTEAUME et $a l ., 2010)$. d) Desarrollar modelos más operativos de la interacción incendio-atmósfera para estimar con más precisión el comportamiento del fuego, particularmente en los Mega-incendios (Clark et al., 2004; CoEn et al., 2010). Necesitamos comprender las condiciones que favorecen su inicio y propagación y la estrategia para limitar su ocurrencia y desarrollo.

e) Mejorar nuestro conocimiento y predicción de las condiciones de inicio y propagación del fuego de copa que sean aplicables a la selvicultura preventiva y a la extinción (CRUZ et al., 2006, 2010; ÁLVAREZ et al., 2012).

f) Incrementar nuestra capacidad de pronóstico del comportamiento del fuego extremo, con especial énfasis en la interacción del fuego con la topografía y los fenómenos de fuego eruptivo (e.g. VIEGAS et al., 2006).

$\mathrm{Si}$ existe un problema relativo a los incendios forestales donde es preciso una aproximación interdisciplinar, con datos generados en laboratorio y campo, ese es el del comportamiento del fuego. Además existe una significativa necesidad de disponer de información de campo procedente de los propios incendios. Y de experimentos ad-hoc que permitan testar, validar y mejorar los modelos de predicción del comportamiento del fuego. Se esté avanzando en el análisis retrospectivo de los incendios, incluyendo el cartografiado de su desarrollo, y ligándolo con las condiciones ambientales durante el fuego (CASTELlNoU, 2010; LÁZARO, 2010). Sin duda ello ayudará a la catalogación de los incendios y una mejor comprensión de su comportamiento en situaciones futuras semejantes. Pero no es suficiente. Hace falta una doble aproximación. Por un lado, se requiere una recogida de información, lo más detallada posible, del comportamiento del fuego durante el incendio. Por otro lado, es preciso efectuar experimentos de campo con fuego real que suministren el nivel de información precisa para avanzar en la elaboración y validación de modelos de predicción del comportamiento del fuego, más realistas y fiables que los actuales. Esta experimentación es absolutamente necesaria y está tropezando con dificultades aparentemente insalvables. La alternativa es disponer de modelos más o menos teóricos que pueden conducir a decisiones erróneas por falta de validación. 
Esperar a los incendios para ver como trabajan los modelos es una decisión inadecuada al problema que entraña bastantes riesgos. Lamentablemente, estamos apagando miles de fuegos, cuya extinción es muy costosa, tenemos una superficie afectada cada año muy considerable y no estamos aprendiendo de todo ello lo suficiente para hacer más eficaz, más eficiente y más seguro el control de los fuegos.

\section{TECNOLOGÍAS DE LA EXTINCIÓN}

Hay mucho margen para las mejoras tecnológicas en el combate de incendios. Baste solo recordar el relativo poco tiempo que llevan operando las brigadas helitransportadas y el decisivo paso que ello ha supuesto en los tiempos de respuesta, llegada al fuego y control de los incendios en su fase inicial. Las estrategias y tácticas, logística y organización del empleo de medios en el combate, la ergonomía y las condiciones de seguridad del personal, son algunos de los temas que requieren mejoras. Por su importancia y relación con la seguridad de los combatientes el empleo del contrafuego exige una atención especial, teniendo en cuenta además la escasez de información científica y técnica sobre él (CASTEllnou et al., 2010; Miralles et al., 2010; DUPUY et $a l .$, 2011; VEGA et $a l .$, 2012). Por otro lado, la modelización de la influencia de las acciones de supresión sobre el comportamiento del fuego y la optimización del empleo de medios extinción no está actualmente desarrolladas de forma adecuada (MARTín et al., 2002).

Muchas de estas cuestiones están a medio camino entre la investigación científica y los desarrollos tecnológicos, en un campo interesante para la colaboración entre la investigación e innovación científica y empresarial, de la que tan necesitada estamos en nuestro país.

\section{RIESGO DE INCENDIO}

La evaluación del riesgo, una parte esencial de la prevención de incendios, ha experimentado en los últimos años una gran transformación, pareja al desarrollo de los SIG y las investigaciones en otros campos del fuego. Se ha pasado así de una estimación del riesgo de base principalmente meteorológica (CAMIA et $a l ., 2003$ ) a unos sistemas mas complejos que incluyen mas factores. Las nuevas propuestas para esa evaluación (Chuvieco et al., 2010; Chuvieco, 2010) la hacen descansar sobre dos componentes: uno relativo a la ignición y propagación potencial del incendio y otro expresivo de su daño potencial. En el primer componente se han incorporado factores físicos y humanos (CHUVIECO et $a l$., 2003). Respecto a las fuentes de ignición, se han considerado la influencia del al cambio de uso en el territorio, y la ocurrencia de fuegos de origen humano (CHOU et al., 1993; Vega-GarCía, 1995; Chuvieco et al., 2003; MARTínez et al., 2009; VILAR DEL HoYo et al., 2011) o por rayos (DíAZ-Avalos et al., 2001; WotTon \& MARTELl, 2005). También se han introducido la estimación de la humedad de los combustibles vivos por sensores remotos (CHUVIECO et al., 2004a,b; AGUADO et $a l ., 2007)$ y la propagación potencial del fuego con la utilización de modelos de predicción de comportamiento del fuego (CHUVIECO et $a l ., 2010)$. Un aspecto particularmente interesante ha sido la consideración explicita de la vulnerabilidad del fuego, como expresión del daño causado por el incendio en su triple vertiente socioeconómica, de degradación potencial de la vegetación y el suelo y la alteración de las calidad intrínseca del paisaje (RODRÍGUEZ Y SILVA et al., 2007; AllozA et al., 2006; MARTíNEZ-VEGA, 2003, 2007: ARRIAZA et al., 2004).

Estas aproximaciones más globales a la evaluación del riesgo tienen la ventaja de poder ofrecer una visión mas completa del problema y generar herramientas de planificación geográfica útiles a los gestores territoriales de recursos. No obstante, plantea problemas de una adecuada ponderación e integración de los cada vez mayor número de componentes de esa visión del riesgo. También necesitan ser adaptados a diferentes escalas temporales y espaciales y definir un marco mas consistente de estimación de la precisión tanto de las variables de entrada como de los resultados finales. Se echa en falta también un mayor esfuerzo de homogeneización de los sistemas de evaluación del riesgo, definido por términos y componentes diferentes en las aproximaciones propuestas por los distintos autores, países y regiones. Esto ayudaría a una 
comparación más objetiva del riesgo entre zonas distintas, facilitando la planificación de medidas de protección y la justificación de los criterios de asignación de recursos de protección.

\section{ACCIONES DE MITIGACIÓN DE RIESGOS POST-INCENDIO Y RECUPERACIÓN DE ÁREAS QUEMADAS}

En muchas zonas ardidas, con pendientes pronunciadas, existe el riesgo de degradación del suelo por erosión y de que se produzcan daños fuera del área quemada por riadas causadas por el aumento de la escorrentía y caudal sólido generado en el área quemada. Estas situaciones son particularmente dramáticas en áreas del mediterráneo y fachada atlántica debido a las fuertes lluvias de otoño. La estabilización de emergencia de los suelos quemados, supone un desafío para los gestores forestales ya que se precisan tomar decisiones en muy poco tiempo para minimizar los riesgos indicados. (FERnÁNDEZ et $a l$., 2011; FontURBEL et $a l$., 2012) Se requiere para ello disponer de recomendaciones y procedimientos que faciliten la priorización de las áreas a ser intervenidas con urgencia después del incendio, ayudándo también a elegir los tratamientos más adecuados (VEGA et $a l$., en prensa). Asociado con este problema se encuentra la evaluación de la severidad del fuego tras incendio ya que este factor es un elemento clave en la priorización de esas actuaciones mitigadoras de urgencia. Aunque se dispone para ello de herramientas basadas en la teledetección y en inventarios de campo, es preciso contar con instrumentos más operativos y fiables que los actuales.

También las tareas de recuperación de las zonas ardidas, a medio y largo plazo, requieren de métodos de recogida de información y de guías que orienten a los gestores en esas labores y su planificación (Alloza et al., 2006). Estas actividades pueden ofrecer oportunidades para el cambio de tipo de bosque, con una modificación de la cubierta vegetal y su estructura que facilite una mayor resistencia y resiliencia frente a nuevos incendios.

En otros casos, ese cambio de especie tras incendios no es factible o recomendable, siendo necesario atender a otros problemas como la predicción de supervivencia del arbolado afectado (VEGA et al., 2011), el efecto de la extracción del arbolado quemado (FERNÁNDEZ et al., 2007) en el suelo y en el regenerado (MADRIGAL et $a l$., 2005; MoyA, et al. 2008) o los tratamientos de apoyo al regenerado (JIMÉNEZ et al.; 2011).

\section{Conclusiones}

Los incendios forestales son fenómenos complejos que no admiten ser catalogados con una visión unidireccional. Como PYNE (2007) señala, hasta ahora venimos corriendo el riesgo de que las aproximaciones física (y técnica), biológica (principalmente ecológica) y social, generadoras de paradigmas aparentemente autosuficientes, alejen la posibilidad de una visión más holística, apoyada en una verdadera interdisciplinariedad (no simplemente multidisciplinariedad). El reto principal es, en línea a lo que sugieren MYERS (2006) y Bowman et al. (2009) integrar esas visiones de forma más armónica para que el papel del fuego en nuestros ecosistemas sea reconocido, aceptado y utilizado, al propio tiempo que esos mismos ecosistemas no se deterioren y puedan también suministrar recursos bienes y servicios al hombre. No sabemos si, contradiciendo al filósofo Bachelard, el hombre alguna vez podrá ver al fuego de forma objetiva, pero a día de hoy la combinación de investigación, análisis y aprendizaje de los hechos observados y junto a una gestión adaptativa inteligente parecen una vía relativamente razonable para intentarlo.

\section{BIBLIOGRAFÍA}

Agee, J.K. \& Lolley, M.R.; 2006 Thinning and prescribed fire effects on fuels and potential fire behavior in an Eastern Cascades forest, Washington, USA. Fire Ecology 2: 3-19.

Ager, A.A.; Finney, M.A.; Mcmahan, A. \& CATHCART, J.; 2010 Measuring the effect of fuel treatments on forest carbon using landscape risk analysis. Natural Hazards and Earth System Science 10(12): 2515-2526.

Aguado, I.; Chuvieco, E.; Boren, R. \& Nieto, H.; 2007. Estimation of dead fuel moisture 
content from meteorological data in Mediterranean areas. Applications in fire danger assessment. Int. J. Wildland Fire 16: 390-397.

Alcamo, J.; Moreno, J.M.; Nováky, B.; Bindi, M.; Corobov, R.; Devoy, R.J.N. et al.; 2007. Europe. In: M.L. Parry, O.F. Canziani, J.P. Palutikof, P.J. van der Linden \& C.E. Hanson (eds.), Climate change 2007: Impacts, adaptation and vulnerability. Contribution of Working Group II to the Fourth Assessment Report of the Intergovernmental Panel on Climate Change: 541-580. Cambridge University Press. Cambridge.

ALLEN, C.D.; 2002. Lots of lightning and plenty of people: An ecological history of fire in the upland Southwest. In: T.R. Vale (ed.), Fire, Native Peoples, and the Natural Landscape: 143-193. Island Press. Covelo.

Alloza, J.A.; Baeza, M.J.; De La Riva, J.; Duguy, B.; EChEVERríA, M.T.; IbARRA, P.; Llovet, J.; Pérez Cabello, F; Rovira, P. \& VALLEJO, V.R.; 2006. A model to evaluate the ecological vulnerability to forest fires in Mediterranean ecosystem. Forest Ecol. Manage. 234: S203.

Álvarez, A.; Gracia, M. \& Retana, J.; 2012. Fuel types and crown fire potential in Pinus halepensis forests. Eur. J. Forest Res. 131: 463-474.

Anav, A. \& Mariotti, A.; 2011. Sensitivity of natural vegetation to climate change in the Euro-Mediterranean area. Climate Research 46: 277-292.

ARChibald, S.; Roy, D.P.; VANwilgen, B.W. \& SCHOLES, R.J.; 2009. What limits fire? An examination of drivers of burnt area in Southern Africa. Global Change Biol. 15: 613-630.

Arriaza, M.; Canas-Ortega, J.F.; CanasMAdueÑo, J.A. \& Ruiz-Avilés, P.; 2004. Assessing the visual quality of rural landscapes. Landscape and Urban Planning 69: 115-125.

Bowman, D.M.; CARLSON, J.M.; Cochrane, M.S.A.; D'ANTONIO, C.M.; Defries, R.S.; DOYLE, J.C.; HARRISON, S.P.; JOHNSTON, F.H.; KeEley, J.E.; KrawchuK, M.A.; Kull, C.A.; Marston,J.B.; Moritz, M.A.; Prentice, I.C.;
Roos, C.I.; Scott, A.C.; Swetnam, T.W.; VAN Der Werf,G.R. \& Pyne, S.J.; 2009. Fire in the Earth system. Science 324, 481-484.

BurRows, N.D.; 2008. Linking fire ecology and fire management in south-west Australian forest landscapes. Forest Ecol. Manage. 255: 2394-2406.

BIROT, Y.; 2009. Convivir con los incendios forestales: lo que nos revela la ciencia. EFI Discussion Paper 15: 1-79.

CABAllero, D.; 2004. Conclusions of the Third WARM Workshop on Forest Fires in the Wildland-urban Interface in Europe. Madrid, Spain, 26-27th of May.WARM Project Final Report. European Commission.

CAmia, A.; VAlera, V.; Marzano, R. \& ETCHIFIDIS, G.; 2003. Spatial Analysis in European Wildland-urban Interface Environment Using GIS. WARM project, http://www.fria.gr/chapters/warmCh21Cami a.pdf.

Camia, A.; Leblon, B.; Cruz, M.; Carlson, J.D. \& AguAdO, I.; 2003. Methods used to estimate moisture content of dead wildland fuels. In: E. Chuvieco (ed.), Wildland Fire Danger Estimation and Mapping. The Role of Remote Sensing Data: 91-117. World Scientific Publishing. Singapore.

Carvalho, A., Flannigan, M.D., Logan, K.A., Gowman, L.M., Miranda, A. \& Borrego, C.; 2010. The impact of spatial resolution on area burned and fire occurrence projections in Portugal under climate change. Climatic Change 98: 177-197.

CARY, G.J.; 1997. FIRESCAPE e a model for simulation theoretical long-term fire regimes in topographically complex landscapes. Australian Bushfire Conference. Bushfire.

Cary, G.J.; Flannigan, M.D.; Keane, R.E.; BRADSTOCK, R.A.; DaVIES, I.D.; LenIHAN, J.M.; LI,C.; LOGAN, K.A. \& PARSONS, R.A.; 2009. Relative importance of fuel management, ignition management and weather for area burned: evidence from five landscapefire succession models. Int. J. Wildland Fire 18(2): 147-156

Castellnou, M.; Kraus, D.; Miralles , M. \& Delogug; 2010. Suppression fire use in learning organizations. In: J.S. Silva, F. Rego, P. Fernandes \& E. Rigolot (eds.), Towards 
Integrated Fire Management - Outcomes of the European Project Fire Paradox.

Castellnou, M.; 2010. Experiencias adquiridas de los grandes incendios 2007-2009. En Taller sobre lecciones aprendidas en grandes incendios forestales. S.E.C.F. Córdoba.

Coen, J.L. \& RigGan, P.J.; 2010. Landscape-Scale Wildland Fire Modeling: Research \& Applications. In: Proc. Australasian Fire Authorities Council and Bushfire Cooperative Research Centre Conf:: 1-11. CD-ROM. Australasian Fire Authorities Council. Darwin,

Conard, S.; Hartzell, T.; Hilbruner, M. \& Zimmerman, G.; 2001. Changing fuel management strategies-The challenge of meeting new information and analysis needs. Int. J. Wildland Fire 10: 267-275.

Chou, Y.H.; Minnich, R.A. \& Chase, R.A.; 1993. Mapping probability of fire occurrence in San Jacinto Mountains, California, USA. Environmental Management 17: 129140.

Chuvieco, E.; Allgöwer, B. \& Salas, F.J.; 2003. Integration of physical and human factors in fire danger assessment. In: E. Chuvieco (ed.), Wildland Fire Danger Estimationand Mapping. The Role of Remote Sensing Data: 197-218. World Scientific Publishing. Singapore

Chuvieco, E.; Aguado, I. \& Dimitrakopoulos, A.; 2004a. Conversion of fuel moisture content values to ignition potential for integrated fire danger assessment. Can. J. Forest Res. 34(11): 2284-2293.

Chuvieco, E.,; Cocero, D.; Riano, D.; Martín, M.P.; Martínez-Vega, J.; De La Riva, J. \& PÉrEZ, F.; 2004b. Combining NDVI and surface temperature for the estimation of live fuel moisture content in forest fire danger rating. RSE 92: 322-331.

Chuvieco, E.; Aguado, I.; Yebra, M.; Nieto, H.; SAlas, J.; Martín, M.P.; VIllar, L.; Martínez, J.; Martín, S.; IBARRA, P.; De LA Riva, J.; BAEZA, J.; RodríGUEZ, F.; MOLINA, J.R.; HerRerA, M.A. \& ZAMORA, R.; 2010 Development of a framework for fire risk assessment using remote sensing and geographic information system technologies. Ecol. Model. 221: 46-58.
CHuvieco, E.; 2010. Integrating spatial data for comprehensive fire risk information systems. VI International Conference on Forest Fire Research (Edited by Domingos Xavier Viegas).

Clark, T.L., Coen, J. \& Latham, D.; 2004: Description of a coupled atmosphere-fire 986 model. Int. J. Wildland Fire 13: 49-64.

Clements, C.; 2011. Effects of complex terrain on extreme fire behavior. In: Synthesis of Knowledge of extreme fire behavior: volume I for fire managers. PNW-GTR-854: 5-22. Forest Service. USDA.

Cruz, M.G., Butler, B.W. \& Alexander, M.E., 2006. Predicting the ignition of crown fuels above a spreading surface fire. Part II: model behavior and evaluation. Int. J. Wildland Fire 15: 61-72.

Cruz, M.G. \& AleXander, M.E., 2010. Assessing crown fire potential in coniferous forests of western North America: a critique of current approaches and recent simulation studies. Int. J. Wildland Fire 19: 377-398.

Conedera, C.;Torriani, D.; NefF, C.; Ricotta, C.; Bajocco, S. \& Pezzatti, G.B.; 2011. Using Monte Carlo simulations to estimate relative fire ignition danger in a low-to medium fire-prone region. Forest Ecol. Manage. 261: 2179-2187.

Díaz-Avalos, C.; Peterson, D.L.; Alvarado, E.; Ferguson, S.A. \& Besag, J.E.; 2001. Space-time modelling of lightning-caused ignitions in the Blue Mountains, Oregon. Can. J. Forest Res. 31: 1579-1593.

Diaz-Delgado, R.; Lloret, F. \& Pons, X.; 2004. Spatial patterns of fire occurrence in Catalonia, NE, Spain. Landscape Ecology 19(7): 731-745.

Donovan, G.H. \& Rideout, D.B.; 2003, An Integer Programming Model to Optimize Resource Allocation for Wildfire Containment. For. Sci. 49: 331-335.

Duguy, B.; Alloza, J.A.; Roder, A.; Vallejo, R. \& PASTOR, F.; 2007. Modelling the effects of landscape fuel treatments on fire growth and behaviour in a Mediterranean landscape (eastern Spain). Int. J. Wildland Fire 16: 619-632.

DupuY, J.L.; LinN, R.R.; Konovalov, V.; Pimont, F.; VegA, J.A. \& JimÉneZ, E.; 2011. 
Exploring three-dimensional coupled fire-atmosphere interactions downwind of wind-driven surface fires and their influence on backfiring using the HIGRAD-FIRETEC model. Int. J. Wildland Fire 20: 734-750. doi:10.1071/WF10035

Dury, M.; Hambuckers, A.; Warnant, P.; Henrot, A.; Favre, E.; Ouberdous, M. et $a l . ; 2011$. Responses of European forest ecosystems to $21 \mathrm{st}$ century climate: Assessing changes in interannual variability and fire intensity. iForest 4: 82-99.

Erdody, T.L., \& Moskal, L.M.; 2010. Fusion of LiDAR and imagery for estimating forest canopy fuels. Remote Sensing of Environment 114: 725-737.

Etienne, M.; Dzerko, M. \& Rigolot, E.; 1996. Browse impact in silvopastoral systems participating in fire prevention in the French Mediterranean region. In: M. Etienne (ed.), Western European Silvopastoral Systems: 93-102. INRA Editions. Paris.

FAO; 2007. Fire Management-Global Assessment 2006. A Thematic Study Prepared in the Framework of the Global Forest Resources Assessment 2005. FAO. Rome.

FERNANDES, P.M.; 2008. Forest fires in Galicia (Spain): the outcome of unbalanced fire management. J. Forest Econ. 14: 155-157.

FERNANDES, P.M.; 2013. Fire-smart management of forest landscapes in the Mediterranean basin under global change. Lanscape and Urban Planning 110: 175-182.

Fernandes, P.; Luz, A. \& Loureiro, C.; 2010. Changes in wildfire severity from maritime pine woodland to contiguous forest types in the mountains of northwestern Portugal. Forest Ecol. Manage. 260: 883-892.

Fernández, C.; Vega, J.A.; Fonturbel, T., Pérez-Gorostiaga， P.; Jiménez， E. \& MADRIGAL, J.; 2007. Effects of wildfire, salvage logging and slash treatments on soil degradation. Land Degradation \& Development 18: 591-607.

FERnÁNDEZ, C.; VeGA, J.A.; JimÉNEZ, E. \& FonTURBEL, T.; 2011. Effectiveness of tree post-fire treatments at reducing soil erosion in Galicia (NW Spain) Int. J. Wildland Fire 20: 104-114

Finney, M.A.; Seli, R.C.; Mchugh, C.W.; Ager, A.A.; BAhro, B. \& AgeE, J.K.; 2007.
Simulation of long-term landscape-level fuel treatment effects on large wildfires. Int. J. Wildland Fire.

Flannigan, M.D.; KrawchuK, M.A.; De Groot, W.J.; WotTon, B.M. \& Gowman, L.M.; 2009. Implications of changing climate for global wildland fire. Int. J. Wildland Fire 18: 483-507.

Fontúrbel, M.T.; VegA, J.A.; PÉrezGorostiaga, P.; Fernández, C.; Alonso, M.; CuIÑAs, P. \& JiMÉNEZ, E.; 2011. Effects of soil burn severity on germination and initial establishment of maritime pine seedlings, under greenhouse conditions, in two contrasting experimentally burned soils. Int. J. Wildland Fire 20: 209-222.

Fontúrbel, M.T.; BARREIRO, A.; VeGA, J.A.; Martín, A.; JimÉnez, E.; CARballas, T.; FERnÁNDEZ, C. \& DíAZ-RAViÑA, M.; 2012. Effects of an experimental fire and post-fire stabilization treatments on soil microbial communities Geoderma 191: 51-60

GALIANA, L.; 2012. Las interfaces urbano-forestales: un nuevo territorio de riesgo en España. Bol. Asoc. Geograf. Esp. 58: 205-226.

Galiana, L.; Herrero, G. \& Solana, J; 2007. Caracterización y clasificación de Interfaces Urbano-Forestales mediante análisis paisajístico. El ejemplo de Sierra Calderota (Comunidad Valenciana, España). In: Proceedings of the Wildfire 2007-4 th International Wildland Fire Conference: 1-13. Sevilla. Spain.

Ganteaume, A.; LAMPIM-Maillet, C.; Guijarro, M.; Hernando, H.; JapPiot, M.; Fonturbel, T.; Pérez-Gorostiaga, P. \& VEGA, J.A.; 2009. Spot fires: fuel bed flammability and capability of firebrands to ignite fuel beds. Int. J. Wildland Fire 18: 951-969.

GILL, A.M.; 2005. Landscape fires as social disasters. An overview of the bushfire proble. Environmental Hazards 6: 65-80.

GiLl, A.M. \& STEPHENS, S.L.; 2009. Scientific and social challenges for the managementof fire-prone wildland-urban interfaces. Environmental Research Letters 4: 034014.

GonZALEZ-CABAN, A.; 2007. Wildland Fire Management Police and Fire Management Economic Efficiency in the USDA Forest Service. In: Proceedings of the Wildfire 2007-4 ${ }^{\text {th }}$ International Wildland Fire Conference. Sevilla. Spain. 
Guijarro, M.; Hernando, C.; Diez, C.; Martínez, E.; MAdRIGAL, J.; LAMPINCabaret,c.; Blanc, P.; Colin, P.Y.; PérezGorostiaga, P.; Vega, J.A. \& Fontúrbel, M.T.; 2002. Flammability of some fuel beds common in the South-European ecosystems. In: D.X. Viegas (ed.), Forest Fire Research \& Wildland Fire Safety. Millpress. Rotherdam.

Guijarro, M.; Pérez-Gorostiaga, P.; Díez Galilea, C.; Hernando, C.; Vega, J. A.; Martínez Herranz, E.; Fonturbel, T. Y MADRIGAL, J.; 2004. SALTUS: proyecto de investigación europeo para el estudio de los saltos de fuego. Montes 76: 13-21.

Gracia, C.; Gil, L. \& Montero, G.; 2005. Impactos sobre el sector forestal. En: J.M. Moreno (coord.), Evaluación Preliminar de los Impactos en España por Efecto del Cambio Climático: 399-435. Ministerio de Medio Ambiente. Madrid.

Hernando, C; Planelles, R.; Ortega, M.; Garrido, S.; Madrigal, J.; GuiJarro, M.; Aragonés, J.I. \& Sebastián, A.; 2012. La opinión de los gestores e investigadores sobre la prevención de incendios forestales en España: resultados del cuestionario "FIRESMART". Montes, 109: 33-38.

Hessl, A.E.; 2011. Pathways for climate change effects on fire: models, data and uncertainties. Progres in Physical geography 35: 393-407.

HiRsch, K.; KAFKA, V.: TyMSTRA, C.; MCAlPine, R.; Hawkes, B.; Stegehuis, H. et al.; 2001. Fire-smart forest management: A pragmatic approach to sustainable forest management in fire-dominated ecosystems. The Forestry Chronicle 77: 1-7.

Hurteau, M.D.; Stoddard, M.T., \& Fulé, P.Z.; 2011. The carbon costs of mitigating highseverity wildfire in southwestern ponderosa pine. Global Change Biology 17: 1516-1521.

JiMÉNEZ, E.; VeGA, J.A.; FERNÁNDEZ, C. \& FONTURBEL, T.; 2011. Is pre-commercial thinning compatible with carbon sequestration? A case study in a maritime pine stand in northwestern Spain. Forestry 84(2): 149-155.

JoHnSOn, E.A.; 1992. Fire and the vegetation dynamics: Studies from the North American boreal forest. Cambridge Studies in Ecology. Cambridge University Press. Cambridge.
Keane, R.E.; Holsinger, L.M.; Pratt, S.D.; 2006. Simulating historical landscape dynamics using the landscape fire succession model LANDSUM version 4.0. Gen. Tech. Rep. No. RMRS-GTR-171CD.

Keeley, J. \& Zedler, P.; 2009. Large, highintensity fire events in southern California shrublands: Debunking the fine-grain age patch model. Ecol. Appl. 19: 69-94.

Keeley, J.E.; Fotheringham, C.J. \& Morais, M.; 1999. Reexamining fire suppression impacts on brushland fire regimes. Science 284: 1829-1832.

KraWchuK, M. \& Moritz, M.; 2011. Constraints on global fire activity vary across a resource gradient. Ecology 92: 121-132

LAMPIN, C.; JAPPIOT, M.; BORGNIET, L. \& LONG, M.; 2006. Cartographie des interfaces habitat-forêt: une approche spatiale pour estimer le risque d'incendie de forêt. Revue internationale de geomatique. Eur. J. GIS Spat. analysis 16(3-4): 321-340.

LAMPIN-MAILlET, C.; JAPPIOT, M.; LONG, M.; Morge, D. \& FERRIER, J.P.; 2009. Characterization and mapping of dwelling types for forest fire prevention. Computers. Environment and Urban Systems 33: 224-232.

Lampin-Maillet, C.; Mantzavelas, A.; GaliaNA, L.; JAPPIOT, M.; LONG, M.; HeRrero, G.;Karlsson, O.; IOSSIFINA, A.; Thalia, L. \& ThANASSIS, P.; 2010. Wildland urban interface,fire behaviour and vulnerability: characterization, mapping and assessment. In: J.S. Silva, F. Rego, P. Fernandes \& E. Rigolot (eds.), Towards Integrated Fire Management-Outcomes of the European Project Fire Paradox. European Forest Institute Research Report 23: 71-92. European Forest Institute. Joenssuu.

LAZARO, A.; 2010. Experiencias adquiridas de los grandes incendios 2007-2009. Taller sobre lecciones aprendidas en grandes incendios forestales. S.E.C.F. Córdoba.

LeHMKUhl, J.; KenNedY, M.; FORD, E.; Singleton, P.; GAINES, W. \& Lind, R.; 2007. Seeing the forest for the fuel: Integrating ecological values and fuels management. Forest Ecol. Manage. 246: 73-80.

LI, C.; 2000. Reconstruction of natural fire regimes through ecological modelling. Ecol. Model. 134(2e3): 129-144. 
Lindner, M.; MaroscheK, M.; Netherer, S.; Kremer, A.; Barbati, A.; García-Gonzalo, J.; Rupert S.; Delzon, S.; Corona, P.; Kolstro, ;M.; Manfred, J.; LeXer, J. \& Marchetti, M.; 2010. Climate change impacts, adaptive capacity, and vulnerability of European. Forest ecosystems. Forest Ecol. Manage. 259: 698-709.

LinN, R.; REISNER, J.; COLMAN, J. \& WinTERKAMP, J.; 2002. Studying wildfire behavior using FIRETEC. Int. J. Wildland Fire 11(4): 233246.

LiU, Y.; Stanturf, J. \& Goodrick, S.; 2010. Trends in global wildfire potential in a changing climate. Forest Ecol. Manage. 259: 685-697.

LoepFe, L.; Martínez-Vilalta, J.; Oliveres, J. PiÑOL, J. \& LlORET, F.; 2010 Feedbacks between fuel reduction and landscape homogenisation determine fire regimes in three Mediterranean areas. Forest Ecol. Manage. 259: 2366-2374.

Loepfe, L.; Martínez-Vilalta, J. \& Piñol, J.; 2012. Management alternatives to offset climate change effects on Mediterranean fire regimes in NE Spain. Climatic Change 115: 693-707.

Loepfe L, Martínez-Vilalta J, \& Piñol J.; 2011. An integrative model of human-influenced fire regimes and landscape dynamics. Environ. Model. Software 26: 1028-1040.

Madrigal, J.; Hernando, C.; Martínez, E.; Guijarro, M. \& Diez, C.; 2005. Regeneración post-incendio de Pinus pinaster Ait. En la sierra de Guadarrama. (Sistema Central, España): Modelos descriptivos de los factores influyentes en la densidad inicial y la supervivencia. Inv. Agraria: Sist. Rec. For. 14(1): 36-51.

Marlon, J.R.; Bartlein, P.J.; Carcaillet, C.; Gavin, D.G.; Harrison, S.P.; Higuera, P.E.; Joos, F.; Power, M.J. \& Prentice, I.C.; 2008. Climate and human influences on global biomass burning over the past two millennia. Nat. Geosci. 1: 697-702.

Martín Fernández, S.; Martínez Falero, E. \& PÉREZ, J.M.; 2002. Optimization of resources management in wildfire combat. Environmental Management 30: 336-352.
Martínez-VeGa, J.; MARTín, M.P. \& RoMero, R.; 2003. Valoración del paisaje en la Zona de Especial Protección de Aves Carrizales y Sotos de Aranjuez (Comunidad de Madrid). GeoFocus 3: 1-21.

MARTÍNEZ-VEGA, J.; RoMERo, R. \& ECHAVARRíA, P.; 2007. Valoración paisajística y ecológica de la Comunidad de Madrid: su integración en un índice sintético de riesgo de incendios forestales. Revista de Teledetección 28: 43-60.

Martínez, J.; Vega-García, C. \& Chuvieco, E.; 2009. Human-caused wildfire risk rating for prevention planning in Spain. J. Env. Manage. 90: 1241-1252.

MaVsar, R.; GonZÁlez-CABÁn, A. \& FARRERAS, V.; 2010. The importance of economics in fire management programmes analysis. In: J.S. Silva, F. Rego, P. Fernandes \& E. Rigolot (eds.), Towards Integrated Fire Management-Outcomes of the European Project Fire Paradox. European Forest Institute Research Report 23: 93-103. European Foret Institute. Joenssuu.

Mell, W.E.; Jenkins, M.A.; Gould, J. \& Cheney, P.; 2007. A physics-based approach to modelling grassland fires. Int. J. Wildland Fire 16(1): 1-22.

Mell, W.E.; Manzello, S.L.; Maranghides, A.; ButrY, D. \& ReHM, R.G.; 2010. The wildland-urban interface fire problem - current approaches and research needs. Int. J. Wildland Fire 19: 238-251.

MERCER, D.E.; JefFreY, P.P.; DAVID, T.B. \& JoHN, M.P.; 2007. Evaluating Alternative Prescribed Burning Policies to Reduce Net Economic Damages from Wildfire. Ame. J. Agric. Econ. 89: 63-77.

Millar, C.I.; StePhenson, N.L. \& Stephens, S.L.; 2007. Climate change and forests of the future. Ecol. Appl. 17: 2145-2151

MinNICH, R.A.; 1983. Fire mosaics in southern California andnorthern Baja California. Science 219: 1287-1294.

MinNICH, R.A.; 2001. An integrated model of two fire regimes. Cons. Biol. 15: 1549-1553.

Minnich, R. \& ChOU, Y.; 1997. Wildland fire patch dynamics in the chaparral of Southern California and Northern Baja California. Int. J. Wildland Fire 7: 221-248. 
Miralles, M.; Kraus, D.; Molina, D.; Loureiro, C.; Delogu, G.; Ribet, N. \& VILALTA, O.; 2010. Overview of suppression fire policies and practices in Europe. In: J.S. Silva, F. Rego, P. Fernandes \& E. Rigolot (eds.), Towards Integrated Fire Management-Outcomes of the European Project Fire Paradox. European Forest Institute Research Report 23: 203-215. European Forest Institute. Joenssuu.

Moghaddas, J.J.; Collins, B.M.; Menning, K.; Moghaddas, E.E. \& STEPHENS, S.L.; 2010. Fuel treatment effects on modelled landscapelevel fire behavior in the northern Sierra Nevada. Can. J. Forest Res. 40(9): 1751-1765.

Molina, J.R.; Herrera, M.A.; ZAMORA, R.; RodríGUEZ y Silva F. \& GonZÁlEZ-CABÁN, A.; 2011. Economic losses to Iberian Swine production from forest fires. Forest Policy and Economics 13: 614-621.

Molina, J.R.; RodrígueZ y Silva, F.; Herrera, M.A. \& ZAMORA, R.; 2009. A simulation tool for socio-economic planning on forest fire suppression management. In: Forest Fires: Detection, Supression and Prevention. Nova Science Publishers. USA.

Moreira, F.; VIEDMA, O.; ARIANOUTSOU, M.; Curt, T.; Koutsias, N.; Rigolot, E. et al.; 2011. Landscape-wildfire interactions in southern Europe: Implications for landscape management. J. Env. Manage. 92: 2389-2402.

MoRenO, J.M.; 2005. Riesgo de incendios forestales. En: J.M. Moreno (coord.), Evaluación Preliminar de los Impactos en España por Efecto del Cambio Climático: 581-615. Ministerio de Medio Ambiente. Madrid.

Moreno, J.M.; Zavala, G.; Martín, M. \& Millán, A.; 2010. Forest fire risk in Spain under future climate change. In: J. Settele, L.D. Penev, T.A. Georgiev, R .Grabaum, V. Grobelnik, V. Hammen, S. Klotz, M. Kotarac \& I. Kuehn (eds.), Atlas of Biodiversity Risks: 72-73. Pensoft. Sofia, Bulgaria.

Moreno, J.M.; Viedma, O.; Zavala, G. \& LuNA, B.; 2011. Landscape variables influencing forest fires in central Spain. Int. J. Wildland Fire 20: 678-689

Moreno, M.V. \& Chuvieco, E.; 2012 Characterisings fire regimes In Spain from statistics. Int. J. Wildland Fire. http://dx.doi.org/10.1071/WF12061.

Moriondo, M.; GOOD, P.; DURÃO, R.; BINDI, M.; Giannakopoulos, C. \& Corte-Real, J.; 2006. Potential impact of climate change on fire risk in the Mediterranean area. Climate Research 31: 85-95.

Moritz, M.A.; 1997. Analyzing extreme disturbance events: fire in Los Padres National Forest. Ecol. Appl. 7: 1252-1262.

Moritz, M.A. \& STEPHENS, S.L.; 2008. Fire and sustainability: Considerations for California's altered future climate. Climatic Change 87: S265-S271.

Moritz, M.A.; KeEley, J.E.; Johnson, E.A. \& SCHAFFNER, A.A.; 2004. Testing a basic assumption of shrubland fire management: how important is fuel age? Front. Ecol. Env. 2(2): 67-72.

Mouillot, F. \& FiEld, C.B.; 2005. Fire history and the global carbon budget: a $1^{\circ} \mathrm{x} \_1^{\circ}$ fire history reconstruction for the 20th century. Global Change Biology 11(3): 398-420.

Moya, D.; Espelta, J.; LoPez-Serrano, F.; Eugenio, M. \& De Las Heras, J.; 2008. Natural post-fire dynamic and serotiny in 10 years- old Pinus halepensis Mill stands along a geographic gradient. Int. J. Wildland Fire 17: 287-292.

Myers, R.L.; 2006. Living with Fire Sustaining Ecosystems and Livelihoods through Integrated Fire Management. Global Fire Initiative. The Nature Conservancy, Tallahassee.

Pausas, J.G.; Llovet, J.; Rodrigo, A. \& VALLEJO, R.; 2008. Are wildfires a disaster in the Mediterranean basin? - a review. Int. J. Wildland Fire 17: 713-723.

Pausas, J.G. \& Keeley, J.E.; 2009. A burning story: the role of fire in the history of life. BioScience 59: 593-601.

PAusas, J.G. \& FernÁndeZ-Muñoz, S.; 2012. Fire regime changes in the Western Mediterranean basin: From fuel-limited to drought-driven fire regime. Climatic Change 110: 215-226.

Pausas, J. G. \& Paula, S.; 2012. Fuel shapes the fire-climate relationship: Evidence from Mediterranean ecosystems. Global Ecology and Bio- 
geography http://dx.doi.org/10.1111/j.14668238.2012.00769.x

Parsons, R; Mell, W. \& Mccauley, P.; 2011. Linking 3D spatial models of fuels and fire: Effects of spatial heterogeneity on fire behavior. Ecol. Model. 222: 679-691

Pastor, E.; Zarate, L.; Planas, E. \& ARNALDOS, J.; 2003. Mathematical models and calculation systems for the study of wildland fire behaviour. Progress in Energy and Combustion Science 29(2): 139-153.

Perchat, S. \& Rigolot, E.; 2005. (Fuel-breaks affected by large fires in 2003: Fire behaviour and use by fire suppression) Comportement au feu et utilisation par les forces de lutte des coupures de combustible touches par les grands incendies de la saison 2003. Ed. de la Cardère. Morières.

PERERA, A.; 2008. BFOLDS 1.0: a spatial simulation model for exploring large scale fire regimes and succession in boreal forest landscapes. Forest research report No. 152. Ontario Forest Research Institute.

Perry, G.L.W.; 1998. Current approaches to modelling the spread of wildland fire: a review. Progress in Physical Geography 22(2): $222 \mathrm{e} 245$.

Piñol, J.; BEVEN, K.J. \& VIEGAS, D.X.; 2005. Modelling the effect oire-exclusion and prescribed fire on wildfire size in Mediterranean ecosystems. Ecol. Model. 183: 397-409.

Piñol, J.; Castellnou, M. \& Beven, K.J.; 2007. Conditioning uncertainty in ecological models: Assessing the impact of fire management strategies. Ecol. Model. 207: 34-44.

Piñol, J.; Terradas, J. \& Lloret, F.; 1998. Climate warming and wildfire hazard and wildfire occurrence in coastal eastern Spain. Climatic Change 38: 345-357.

Prestemon, J.P.; AbT, K.L. \& BARbour, R.J.; 2012. Quantifying the net economic benefits of mechanical wildfire hazard treatments on timberlands of the western United States. Forest Policy and Economics. 21: 44-53.

Price, O.F. \& Bradstock, R.A.; 2010. The effect of fuel age on the spread of fire in sclerophyll forest in the Sydney region of Australia. Int. J. Wildland Fire 19:35-45.
PueYo, S.; 2007. Self-Organised Criticality and the Response of Wildland Fires to Climate Change. Climate Change 82: 131-161.

Pyne, S.J.; 1982. Fire in America: A cultural history of wildland and rural fire. Princeton Univ. Press. Princeton.

Pyne, S.J.; 1995. World fire. In: The Culture of Fire on Earth. University of Washington Press. Seattle and London.

Pyne, S.; 2007. Problems, paradoxes, paradigms: triangulating fire research. Int. J. Wildland Fire 16: 271-276.

Pyne, S.J., ANDREWs, P.L. \& LAVEN, R.D.; 1996. Introduction to wildland fire (2nd ed.). John Wiley and Sons. New York.

QuAdRENNIAL Fire ReVIEW; 2009. Final Report, January 2009. Available at http://www.nifc.gov/QFR/QFR2009Final.pdf.

RADElOFF, V.C.; HAMMER, R.B.; STEWART, S.I.; FRIED, J.F.; HOLCOMB, S.S. \& MCKEEFRY, J.F.; 2005. The wildland urban interface in the United States. Ecol. Appl. 15(3): 799-805.

ReInHARDT, E.D.; KEANE, R.E.; CALKIN, D.E. \& COHEN, J.D.; 2008. Objectives and considerations for wildland fuel treatment in forested ecosystems of the interior western United States. Forest Ecol. Manage. 256: 1997-2006.

Riaño, D.; Chuvieco, E.; Condes, S.; GonZalez-Matesanz, J. \& Ustin, S.L.; 2004. Generation of crown bulk density for Pinus sylvestris L. from LiDAR. Remote Sensing of Environment 92: 345-352.

RODRIGUEZ Y SILVA, F.; 2009. ECONOSINAMI, una herramienta informática para la evaluación económica de los programas de defensa contra incendios forestales. En: S.E.C.F.Junta de Castilla-León. Actas del $5^{\circ}$ Congreso Forestal Español CD-Rom 5CFE01-466: 2-13. Sociedad Española de Ciencias Forestales. Ávila.

RodríGUEZ y Silva, F.; Molina, J.R.; HerRerA, M. \& ZAMORA, R.; 2007. Vulnerabilidad socioeconómica de los espacios forestales frente al impacto de los incendios, aproximación metodológica mediante sistemas de información geográficos (proyecto Firemap). In: Proceedings of the Wildfire 2007-4 $4^{\text {th }}$ International Wildland Fire Conference. Sevilla. Spain. 
Rodríguez y Silva, F.; González-CabÁn, A. 2010. "SINAMI": a tool for the economic evaluation of forest fire management programs in Mediterranean ecosystems. Int. J. Wildland Fire 19: 927-936.

Rodriguez y Silva, F; Molina Martínez J.; HerRera MachucA, M. \&. Rodriguez LEAL, J.; 2012. Visual-Seveif, una herramienta para la integración de la simulación del comportamiento del fuego y la evaluación económica del impacto de los incendios forestales. In: $I V$ International Fire Economic, Planning and Climate Change. USDA Forest Service. GTR. PSW Station Research. México.

Romero-CAlcerrada, R.; Novillo, C.J.; Millington, J.D.A. \& GómEZ-JiMÉNEZ, I.; 2008. GIS analysis of spatial patterns of human-caused wildfire ignition risk in the SW of Madrid (central Spain) Landscape Ecology 23: 341-354.

Ruiz-Mirazo, J.; Robles, A.B. \& GonzÁlezREBOLLAR, J.L.; 2009. Pastoralism in Natural Parks of Andalusia (Spain): a tool for fire prevention and the naturalization of ecosystems. Options Méditerranéennes, Serie A(91): 141-144.

Ruiz-Mirazo, J.; Robles A.B. \& GonzÁlez REBOLLAR, J.L.; 2011. Two-year evaluation of fuelbreaks grazed by livestock in the wildfire prevention program in Andalusia (Spain). Agriculture, Ecosystems and Environment 141: 13-22.

San-Miguel, J. \& CAMiA, A.; 2009. Forest fires at a glance: facts, figures and trends in the EU. In: Y. Birot (ed.), Living with wildfire: What science can tell us. EFI Discussion Paper 15: 11-18. EFI, Joensuu.

Serrada, R.; Aroca, M.J.; RoIG, S.; Bravo, A. \& GÓMEZ, V.; 2011. Impactos, vulnerabilidad y adaptación al cambio climático en el sector forestal. Ministerio de Medio Ambiente y Medio Rural y Marino. Madrid.

SERRADA, R.;MuÑOZ, I.; MARTÍNEZ, J.; LOPEZ DE DiegO, L.; RodrigueZ, M.; GonZALEZ, J.R.; OCAÑA, L.; CABEZAS, F.; MAGRO, J. \& ROMERO, F.; 2006. Incendios forestales: Tratamiento de superficies quemadas. Congreso Nacional de Medio Ambiente. Madrid.

ShARPLES, J.J.; 2009. An overview of mountain meteorological effects relevant to fire beha- viour and bushfire risk. Int. J. Wildland Fire 18 (7):737-754.

Syphard, A.D.; KeEley, J.E. \& Brennan, T.; 2011. Comparing the role of fuel breaks across southern California national forests. Forest Ecol. Manage. 261: 2038-2048.

Stephens, S.; Millar, C. \& Collins, B.; 2010. Operational approaches to managing forests of the future in Mediterranean regions within a context of changing climates. Environmental Research Letters 5: 024003.

Stockmann, K.; Hyde, K.; Jones, J.; LOOFLER D. \& SiLVERSTEIN, R.; 2010. Integrating fuel treatment into ecosystem management: a proposed project planning process. Int. J. Wildland Fire 19: 725-736.

Sullivan, A.; 2009a. Wildland surface fire spread modelling, 1990-2007. 2: Empirical and quasi-empirical models Int. J. Wildland Fire 18(4): 369-386.

Sullivan, A.; 2009b.Wildland surface fire spread modelling, 1990-2007. 1: Physical and quasi-physical models. Int. J. Wildland Fire 18(4): 349-368.

Sullivan, A. ; 2009c.Wildland surface fire spread modelling, 1990-2007. 3: Simulation and mathematical analogue models. Int. J. Wildland Fire 18(4): 387-403

Thavaud, P. (ed.); 2009. Guide pratique pour l'entretien des coupures de combustible par le pastoralisme. Réseau Coupures de combustible. $n^{\circ} 12$. Editions de la Cardère. Morières.

Thonicke, K.; Spessa, A.; Prentice, I.C.; HARrison, S.P.; Dong, L. \& CARMONAMoreno, C.; 2010. The influence of vegetation, fire spread and fire behaviour on biomass burning and trace gas emissions: Results from a process-based model. Biogeoscience 7: 1991-2011.

Vaillant, N.M.; J. Fites-Kaufman, A.L.; ReIner, E.K.; NoONAN-Wright, S. \& DAILEY, N.; 2009. Effect of fuel treatments onfuels and potential fire behavior in California, USA, national forests. Fire Ecology 5(2): 14-29.

VAZQueZ, A.; PÉreZ, B.; FernándeZ-GonZÁLEZ, F. \& Moreno, J.M.; 2002. Recent fire regime characteristics and potential natural vegetation relationships in Spain. J. Veg. Sci. 13: 663-676. 
VazQuez De La Cueva A.; Del Barrio, J.M.; Quero, M.O. \& Palomares, O.; 2006. Recent fire regime in peninsular Spain in relation to forest potential productivity and population density. Int. J. Wildland Fire 15(3): 397-405.

VazQuez De La Cueva, A.; 2012. Relative Contributions of Forest Vegetation, Land Cover, Topography and Climate in Explaining Fire Regime Patterns (1974-2005) in Peninsular Spain. I International Scholarly Research Network ISRN. Forestry: 1-11. Article ID 479491

VegA, J.A.; FernándeZ, C.; JimÉneZ, E. \& RuIZ, A.D.; 2009a. Evidencias de cambio climático en Galicia a través de la tendencia de los índices de peligro de incendios forestales. En: V. Pérez Muñuzuri, M. Fernández Cañamero e J.L. Gómez Gesteira (coords.), Evidencias e impactos del cambio climático en Galicia: 173-194. Xunta de Galicia. Vigo.

VEGA, J.A.; FERnÁNDEZ, C.; JiMÉNEZ, E. \& RUIZ, A.D.; 2009b. Impacto de un escenario de cambio climático sobre el peligro de incendios en Galicia. En: V. Pérez Muñuzuri, M. Fernández Cañamero e J.L. Gómez Gesteira (coords.), Evidencias e impactos del cambio climático en Galicia: 581-206. Xunta de Galicia. Vigo.

Vega, J.A.; JimÉnez, E.; Vega, D.; Ortiz, L. \& PÉreZ, J.R.; 2011. Pinus pinaster Ait. tree mortality following wildfire in Spain. Forest Ecol. Manage. 261: 2232-2242

VeGA, J.A.; JiMÉNEZ, E.; DuPUY, J. \& LinN, R.; 2012. Effects of flame interaction on the rate of spread of heading and suppression fires in shrubland experimental fires. Int. $J$. Wildland Fire 21(8): 950-960.

Vega, J.A.; Fonturbel, T.; Fernández, C.; Díaz-Raviña, M.; Carballas, T.; Martín, A.; González-Prieto, S.; Merino, A. \& BENITO, E.; 2013. Acciones de emergencia en áreas forestales quemadas: guía y bases para su planificación y ejecución en Galicia. Xunta de Galicia-INIA. Madrid.

Vega-García, C.; WoOdard, T.; AdAMOWICZ \& Lee, B.; 1995. A logit model for predicting the daily occurence of human caused forest fires. Int. J. Wildland Fire 5: 101-111.

Vega-García, C. \& Chuvieco, E.; 2006. Applying local measures of spatial heterogeneity to Landsat-TM Images for predicting wildfire occurrence in Mediterranean landscapes. Landscape Ecology 21: 595-605.

VÉLEZ, R.; 2002: Forest fire prevention with a target: The rural people. In: Proceedings of the IV International Conference on Forest Fire Research. Luso, Portugal.

Vélez, R.; 2005. Community Based Fire Management in Spain Forestry Department. Food and Agriculture Organization of the United Nations. Rome.

VÉLEZ, R.; 2010. Prescribed burning for improved grazing and social fire prevention: the Spanish EPRIF Programme. In: C. Montiel $\&$ D. Kraus (eds.), Best practices of fire useprescribed burning and suppression fire programmes in selected case-study regions in Europe: 107-120. European Forest Institute. Joenssuu.

VIEDMA, O.; 2008. The influence of topography and fire in controlling landscape composition and structure in Sierra de Gredos (Central Spain). Landscape Ecology 23(6): 657-672.

VIEGAS, D.; 2006. Parametric study of an eruptive fire behaviour model. Int. J. Wildland Fire 15: 169-177

VILÉN, T. \& FERNANDES, P.M.; 2011. Forest fires in Mediterranean countries: $\mathrm{CO}_{2}$ emissions and mitigation possibilities through prescribed burning. Environmental Management 48: 558-567.

Weber, R.O.; 1991. Modelling fire spread through fuel beds. Progress in Energy and Combustion Science 17(1): 67-82.

WERTH, P.; 2011. Critical fire weather patterns. In: Synthesis of Knowledge of extreme fire behavior: volume I for fire managers: 25-48. USDA Forest Service, PNW-GTR-854.

Wotton, B.M.; \& MARTEll, D.L.; 2005. A lightning fire occurrence model for Ontario. Can. J. Forest Res. 35: 1389-1401. 